\title{
Assessment and management of a patient with paraneoplastic dermatomyositis
}

\author{
Ioan-Cristian Lupescu', Adriana Octaviana Dulamea ${ }^{1,2}$ \\ ${ }^{1}$ Neurology Clinic, Fundeni Clinical Institute, Bucharest, Romania \\ 2"Carol Davila" University of Medicine and Pharmacy, Bucharest, Romania
}

\begin{abstract}
Dermatomyositis (DM) is an idiopathic inflammatory myopathy, that can be associated with malignancy. We report the case of a 60-years-old woman, diagnosed and treated for left breast cancer, with residual neoplasm following treatment, who was admitted for generalized myalgias and tetraparesis with predominance of paraparesis. Clinical exam revealed heliotrope rash with bilateral palpebral edema and an erythematous eruption on both thighs and anterior thorax. Motor deficits were predominantly proximal. Paraclinical evaluation excluded other etiologies and revealed elevated serum muscle enzymes and electromyographic pattern of myopathy. An MRI was performed and showed diffuse edematous infiltration of skeletal muscles. Symptoms diminished under corticotherapy, intravenous immunoglobulins and cyclophosphamide. Left mastectomy was eventually performed, but DM symptoms reappeared a few months later. Despite extensive search, no recurrence of tumor was found. However, under treatment, the patient once again recovered, and later resumed her activity.
\end{abstract}

Keywords: dermatomyositis, paraneoplastic, myopathy

Abbreviations (in alphabetical order):
ALT - Alanine aminotransferase
AST - Aspartate aminotransferase
CK - Creatine kinase
HCV - Hepatitis C virus
HIV - Human immunodeficiency virus
ICU - Intensive care unit
CK-MB - Creatine kinase muscle brain
LDH - Lactate dehydrogenase
CT - Computer tomography
DTR - Deep tendon reflexes
MRC - Medical Research Council
MRI - Magnetic resonance imaging
HBV - Hepatitis B virus
STIR - Short tau inversion recovery

\section{INTRODUCTION}

Dermatomyositis (DM) is an idiopathic inflammatory myopathy, characterized by symmetrical proximal muscle weakness, various distinctive skin findings and confirmation of muscle inflammation.

Association between DM and cancer is well established, based on epidemiologic studies (1-3), as well as on their temporal relationship (4).

Clinical risk factors for association with malignancy include older age at onset, cutaneous necrosis, cutaneous vasculitis and presence of dysphagia, while arthritis and interstitial lung disease constitute protective factors (5-6).

Recent emphasis has been placed on antibody testing, malignancy being linked with presence of anti-155/140 antibody (7-8) and absence of myositis specific/associated antibodies (anti-Jo-1, antiPM-Scl, anti-U1-RNP, anti-U3-RNP, anti-Ku) (8).

Pathogenesis is incompletely understood, but most likely determined by an immune mechanism directed against tumor antigens and similar antigens expressed in regenerating muscle (9-10). 


\section{CASE PRESENTATION}

A 60-years-old woman was diagnosed with left breast cancer in April 2013 and treated with Docetaxel (7 sessions) and radiation therapy (25 sessions, 2 Gray/session), with residual neoplasm following treatment.

Based on histopathologic examination, the neoplasm was a poorly differentiated (G3) invasive ductal carcinoma, with positive immunohistochemistry for estrogen receptors (99\% of tumor cells), progesterone receptors (20\%), Ki67 (95\%), P53 (99\%) and negative for Her2.

Generalized myalgias and motor deficits started in October 2013, with progressive worsening, leading to DM diagnosis in January 2014. However, symptoms continued under methylprednisolone 32 mg q.d.

The patient was admitted to our department, presenting tetraparesis with predominance of paraparesis.

Clinical findings included fever, bilateral palpebral edema with heliotrope rash and left upper arm edema. An erythematous eruption was observed on both thighs (Holster sign) and anterior thorax.

Neurological exam highlighted tetraparesis with predominant involvement of proximal muscles, globally diminished DTRs and severe dysphagia, for which nasogastric intubation was performed.
TABLE 1. Motor deficits

\begin{tabular}{|l|}
\hline 3/5 MRC proximal le upper limb \\
\hline 4/5 MRC proximal right upper limb \\
\hline 1/5 MRC proximal paraparesis \\
\hline 4/5 MRC distal paraparesis \\
\hline
\end{tabular}

Laboratory findings showed leukocytosis, normochromic normocytic anemia, low serum iron and elevated muscle enzymes.

TABLE 2. Muscle enzymes

\begin{tabular}{|c|c|c|}
\hline Enzyme & Findings & Normal range \\
\hline $\mathrm{CK}$ & $2307 \mathrm{U} / \mathrm{L}$ & $7-171 \mathrm{U} / \mathrm{L}$ \\
\hline $\mathrm{CK}-\mathrm{MB}$ & $55 \mathrm{U} / \mathrm{L}$ & $0-25 \mathrm{U} / \mathrm{L}$ \\
\hline $\mathrm{AST}$ & $319 \mathrm{U} / \mathrm{L}$ & $0-35 \mathrm{U} / \mathrm{L}$ \\
\hline $\mathrm{ALT}$ & $153 \mathrm{U} / \mathrm{L}$ & $0-45 \mathrm{U} / \mathrm{L}$ \\
\hline $\mathrm{LDH}$ & $648 \mathrm{mmol} / \mathrm{L}$ & $135-225 \mathrm{mmol} / \mathrm{L}$ \\
\hline
\end{tabular}

Biomarkers were collected, including autoimmune, infectious (HBV, HCV, HIV, Borrelia), protein electrophoresis and thyroid hormones, all within normal range.

Electromyography confirmed the presence of a predominantly proximal myopathy.

MRI of cervico-thoraco-abdomino-pelvic musculature was performed and revealed edematous infiltration of muscular fibers (high signal on T2-FatSat) without fatty degeneration (high signal on T1), affecting all examined muscles, suggestive for inflammatory myopathy (Fig. 1).

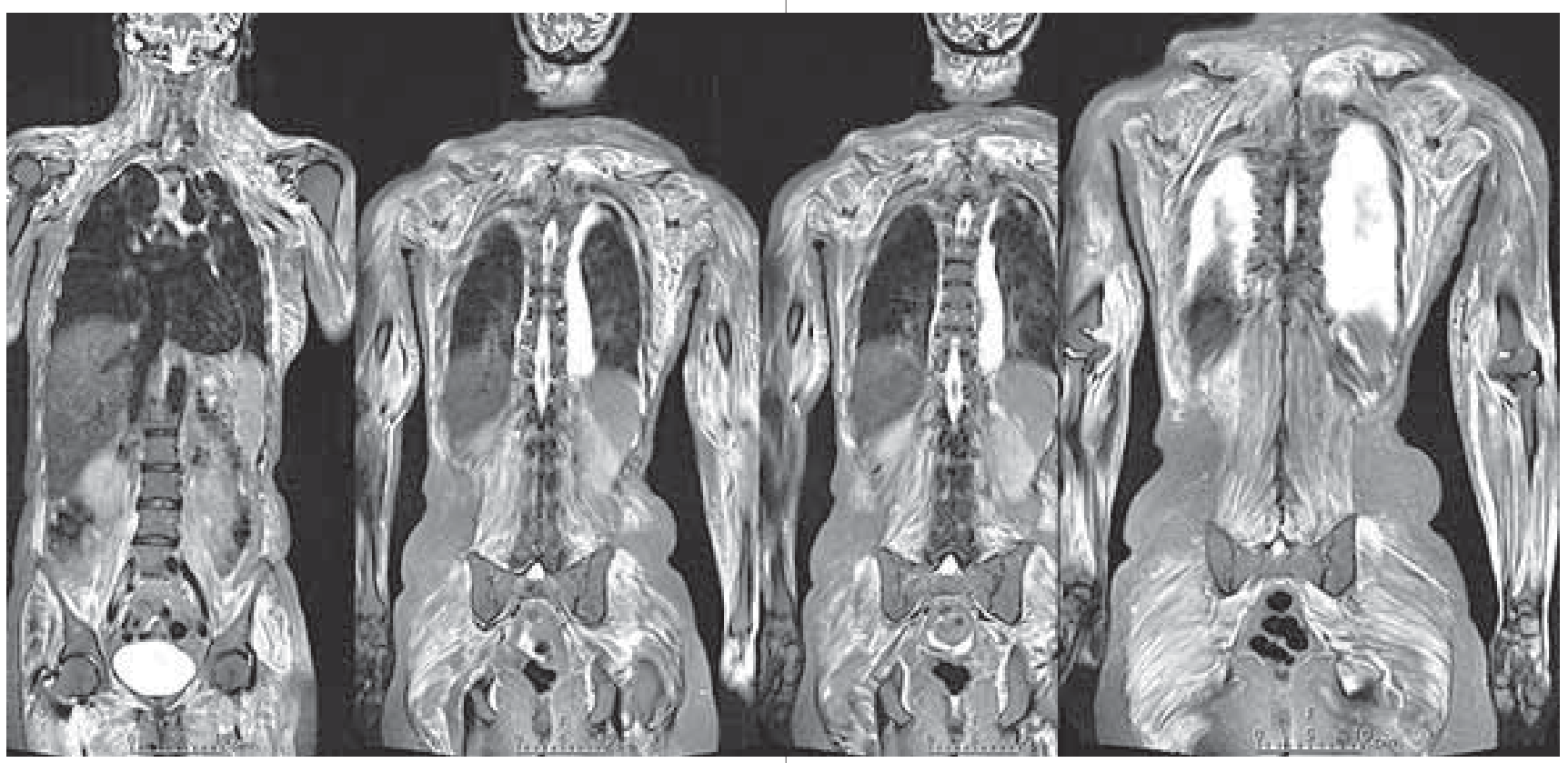

FIGURE 1. MRI evaluation with STIR sequence of body musculature, showing diffuse edematous infiltration of muscles (high-signal intensity) and bilateral pleural effusion. 
Suspecting metastatic disease, a thoraco-abdominal CT scan was done, revealing an inflammatory infiltrate in the left lung and bilateral pleural effusion. Thoracentesis with fluid analysis excluded malignancy.

Under antibiotic therapy, pleural effusions and inflammatory infiltrate diminished significantly.

Immunoglobulins (28 vials in 5 days) and $\mathrm{Cy}-$ clophosphamide (200 mg b.i.d. 4 days) were added to Methylprednisolone (16 mg t.i.d.), with improvement of symptoms.

The patient was discharged with Methylprednisolone $16 \mathrm{mg}$ b.i.d., Alpha-lipoic acid b.i.d. and Gabapentin 300 mg b.i.d..

Residual neoplasm was removed 3 months later through left mastectomy.

Dermatological exam in May 2014 highlighted generalized xerosis and edematous-erythematous plaques on the right hemiface, thighs and calves, recommending continuation of systemic corticotherapy.
In June 2014, muscle symptoms relapsed and the patient was admitted to the ICU. However, no recurrence of the tumor was detected.

MRI of cervical region was performed and revealed the same edematous infiltration affecting all examined muscles (including tongue muscles), albeit diminished by comparison with the previous examination (Fig. 2).

The patient was tested for anti-Jo antibodies, but results came back negative.

Improvement once again occurred with proper treatment and the patient resumed her activity in January 2015.

\section{DISCUSSIONS}

In our case, DM developed after breast cancer diagnosis and treatment, while the patient presented residual neoplasm. No metastases were identified.

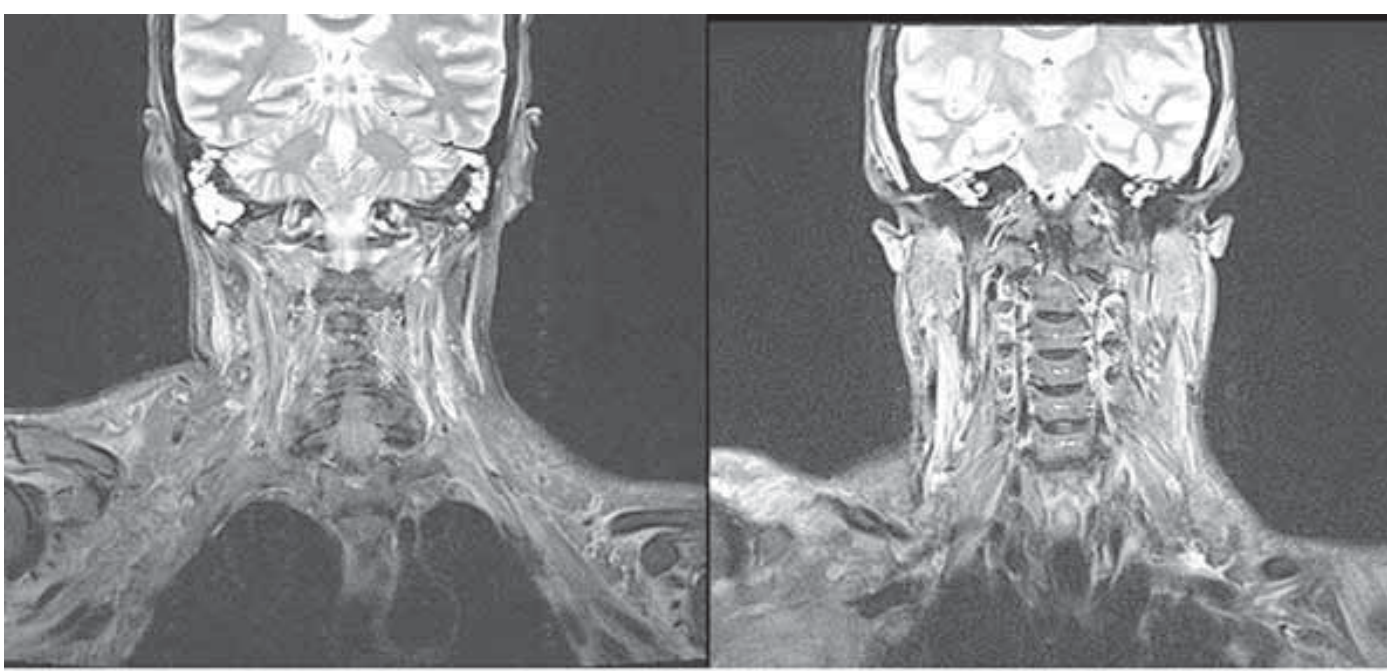

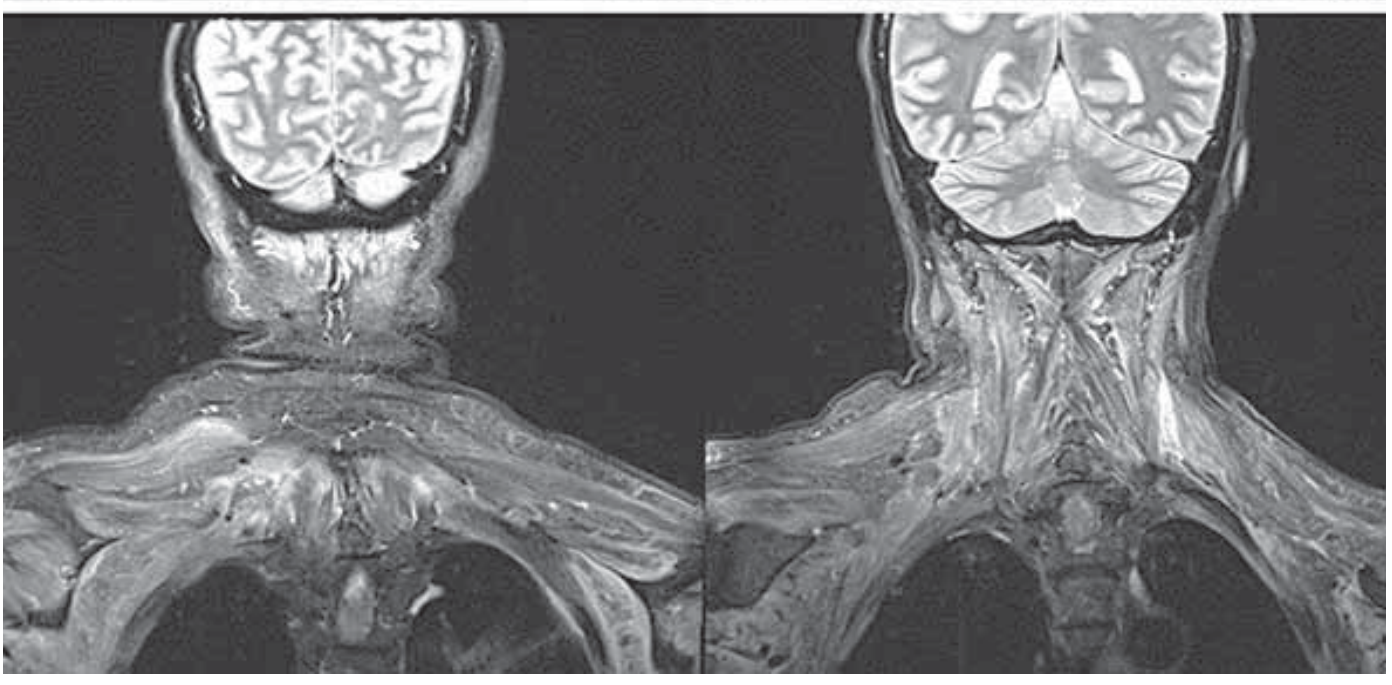

FIGURE 2. MRI evaluation with STIR sequence of cervical musculature, showing the same high-signal intensity, characteristic for edematous infiltration. There is also bilateral mastoiditis. 
In the absence of muscle biopsy, diagnosis was based on clinical picture corroborated with laboratory, electromyographic and imaging signs of muscle involvement, plus exclusion of other causes.

The most widely used diagnostic criteria are the Bohan \& Peter Criteria (1975).

TABLE 3. Bohan \& Peter Criteria

\begin{tabular}{|l|}
\hline 1. Proximal symmetrical muscle weakness \\
\hline 2. Muscle biopsy revealing myosi s \\
\hline 3. Elevated serum levels of skeletal muscle enzymes \\
\hline 4. Electromyographic pa ern of myopathy \\
\hline 5. Typical skin findings of DM \\
\hline
\end{tabular}

"Definite" DM is established if the $5^{\text {th }}$ criterion is present, along with three of the other four (as was in our case). "Probable" and "possible" DM are considered if the $5^{\text {th }}$ criterion is present, along with two of the four and, respectively, one of the four criteria. (11-12)

Specific skin changes associated with DM include the heliotrope rash, Gottron's sign, Gottron's papules and photodistributed erythema or poikiloderma (the shawl and V signs). Clinicians should also look for other dermatological findings, such as nailfold abnormalities, scalp involvement or calcinosis cutis.

Another particular finding is the Holster sign, which consists of an erythematous/violaceous rash on the lateral aspects of thighs. Holster sign was found to be very specific for DM, but under recognized by clinicians other than dermatologists. (13)

Regarding paraclinical evaluation, MRI has the advantage of assessing large areas of muscle and can help in guiding biopsy. Muscle edema intensity using MRI STIR has been shown to correlate with clinical status in the acute phase and to decrease after treatment. (14)

Worth mentioning is the relapse of DM in the absence of tumor recurrence. Unfortunately, no evaluation for antibodies was performed, apart from anti-Jo, which are the most common myositis-specific antibodies. Results came back negative, but that was expected, since they are not associated with paraneoplastic DM.

Better understanding of pathogenesis will be needed to explain the various temporal relationships between malignancy and inflammatory myopathies.
Conflict of interest: none declared Financial support: none declared

\section{REFERENCES}

1. Sigurgeirsson B., Lindelöf B., Edhag O. et al. Risk of cancer in patients with dermatomyositis or polymyositis. A population-based study. N Engl J Med. 1992; 326(6):363-367.

2. Buchbinder R., Forbes A., Hall S. et al. Incidence of malignant disease in biopsy-proven inflammatory myopathy. A population-based cohort study. Ann Intern Med. 2001; 134(12):1087-1095.

3. Chen Y.J., Wu C.Y., Huang Y.L. et al. Cancer risks of dermatomyositis and polymyositis: a nationwide cohort study in Taiwan. Arthritis Res Ther. 2010; 12(2):R70.

4. Zantos D., Zhang Y., Felson D. The overall and temporal association of cancer with polymyositis and dermatomyositis. J Rheumatol. 1994; 21(10):1855-1859.

5. Wang J., Guo G., Chen G. et al. Meta-analysis of the association of dermatomyositis and polymyositis with cancer. Br J Dermatol. 2013; 169(4):838-847.

6. Lu X., Yang H., Shu X. et al. Factors predicting malignancy in patients with polymyositis and dermatomyostis: a systematic review and meta-analysis. PLoS One. 2014; 9(4):e94128.

7. Kaji K., Fujimoto M., Hasegawa M. et al. Identification of a novel autoantibody reactive with 155 and $140 \mathrm{kDa}$ nuclear proteins in

patients with dermatomyositis: an association with malignancy. Rheumatology (Oxford). 2007; 46(1):25-28.

8. Chinoy H., Fertig N., Oddis C.V. et al. The diagnostic utility of myositis autoantibody testing for predicting the risk of cancer-associated myositis. Ann Rheum Dis. 2007; 66(10):1345-1349.

9. Levine S.M. Cancer and myositis: new insights into an old association. Curr Opin Rheumatol. 2006; 18(6):620-624.

10. Dankó K., Ponyi A., Molnar A.P. et al. Paraneoplastic myopathy. Curr Opin Rheumatol. 2009; 21(6):594-598.

11. Bohan A., Peter J.B. Polymyositis and dermatomyositis. N Engl J Med. 1975; 292(7):344-347.

12. Bohan A., Peter J.B. Polymyositis and dermatomyositis. N Engl J Med. 1975; 292(8):403-407.

13. Stephens, Dean H., Schwarzenberger et al. The Holster Sign: A Specific but Under Recognized Skin Finding in Dermatomyositis. Abstr. in: Arthritis Rheum. 2009; 60 Suppl 10:818.

14. Tomasová Studynková J., Charvát F., Jarosová K. et al. The role of MRI in the assessment of polymyositis and dermatomyositis. Rheumatology (Oxford). 2007;46(7):1174-1179. 